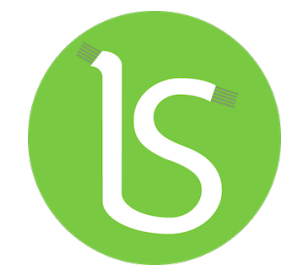

\title{
Estrategias innovadoras de aprendizaje aplicadas en la Experiencia Educativa ¿Cómo aplicar la Estadística en proyectos de investigación?, integradas en las plataformas EMINUS 4 y TEAMS (Universidad Veracruzana)
}

\author{
Diana Donají Del Callejo Canala \\ Margarita Edith Canal Martínezb \\ Mónica Rubiette Hákim Krayemc
}

Resumen - Se presenta la intervención pedagógica-metodológica del diseño de estrategias innovadoras aplicadas a la Experiencia Educativa ¿Cómo aplicar la Estadística a proyectos de Investigación?, perteneciente al Área de Formación de Elección Libre (AFEL), impartida en el periodo escolar enero- febrero 202I. Por primera vez esta intervención fue integrada en ambientes virtuales a través de la plataforma EMINUS 4 de la Universidad Veracruzana y Teams. Los resultados significativos obtenidos, muestran que el $89 \%$ del total de estudiantes acreditaron, evidenciando que son capaces de aplicar y reconocer la transcendencia de la Estadística en proyectos de investigación, contribuyendo a su formación integral con competencias idóneas para insertarse al campo laboral.

Palabras clave - Procesos educativos innovadores, Aprendizaje basado en problemas, Estrategias innovadoras, Enfoque por competencias, Modalidad VirtualEMINUS-Teams.

Abstract - The pedagogical-methodological intervention of the design of innovative strategies applied to the Educational Experience is presented, How to apply Statistics to Research projects? Belonging to the Free Choice Training Area (AFEL), taught in the school period January-February 202I. For the first time this intervention was integrated into virtual environments through the EMINUS 4 platform of the Universidad Veracruzana and Teams. The significant results obtained show that $89 \%$ of all students accredited, evidencing that they are capable of applying and recognizing the importance of Statistics in research projects, contributing to their comprehensive training with suitable skills to enter the labor field.

Keywords - Innovative educational processes, Problem-based learning, Innovative strategies, Competency approach, Virtual-EMINUS-Teams Modality.

\section{CÓMO CITAR \\ HOW TO CITE: \\ Del Callejo Canal, D. D., Canal Martínez, M. E., \& Hákim Krayem, M. R. (2022). Estrategias innovadoras de aprendizaje aplicadas en la Experiencia Educativa ¿Cómo aplicar la Estadística en proyectos de investigación?, integradas en las plataformas EMINUS 4 y TEAMS (Universidad Veracruzana). Interconectando Saberes, (13), 85-99. https://doi.org/ I 0.25009/is.v0i I 3 .2707 \\ Recibido: 31 de mayo de 2021 Aceptado: 22 de noviembre de 2021 \\ Publicado: 31 de enero de 2022}

\footnotetext{
${ }^{a}$ Universidad Veracruzana, México. E-mail: ddelcallejo@uv.mx

b Universidad Veracruzana, México. E-mail: mcanal@uv.mx

c Universidad Veracruzana, México. E-mail: rhakim@uv.mx
} 


\section{INTRODUCCIÓN}

"Si uno considera los cambios como amenaza, nunca innovará. No deseche algo simplemente porque no era lo que planificó, lo inesperado es la mejor fuente de innovación." Drucker (Citado por Mejía, 2000).

La Organización para la Cooperación y Desarrollo Económicos [OCDE] afirma que "uno de los principales objetivos de la educación superior es aportar egresados con las competencias necesarias para lograr el existo en el mercado laboral" (2019, p. 12), en ese tenor el Sistema de Educación Superior de México enfrenta retos importantes respecto a la calidad y la garantía de que los estudiantes desarrollen las competencias relevantes "para contribuir de forma efectiva al desarrollo económico y a la sociedad en su conjunto" (OCDE, 2019, p. 14). Por su parte, la Asociación Nacional de Universidades e Instituciones de Educación Superior de México (ANUIES), menciona que el compromiso que deben asumir las instituciones:

...implica una enorme responsabilidad para contribuir con la mayor pertinencia al desarrollo del país, mediante la formación avanzada de un creciente número de profesionistas, la generación y aplicación innovadora del conocimiento, la difusión de la cultura y la atención a los problemas locales y nacionales. (2018, p.17)

Derivado de lo anterior, las Instituciones de Educación Superior (IES), desde su quehacer social, requieren cambiar el paradigma de sus funciones académicas y de gobernanza institucional tradicionales, para construir y afrontar las necesidades actuales, para potenciar y contribuir al desarrollo local, regional, nacional y visualizarse en un mundo global y con ello, impactar en el desarrollo económico y social del país. Ello implica, sin duda, un cambio en las formas de planear, organizar y ejecutar "las funciones de docencia, investigación -generación y distribución del conocimiento- $y$ extensión y difusión de sus servicios de manera renovada, innovadora y sustentable, con el fin de "reducir la desigualdad social, aumentar la competitividad y erradicar la pobreza que lacera a millones de mexicanos" (ANUIES, 2018, p. 17).

La Universidad Veracruzana (UV), como una IES pública, no ha estado ajena a estos desafíos y cambios de paradigma en sus procesos institucionales, prueba de ello, es la instauración del Modelo Educativo Institucional (MEI) y su enfoque por competencias -entre otras transformaciones-, que concibe al estudiante como centro de su propio aprendizaje, permitiendo que éstos desarrollen competencias teóricas, heurísticas y axiológicas que les permitan insertarse al mundo laboral con capacidades disciplinarias y aprendizajes significativos que generen nuevas formas de ser - hacer y vivir en sociedad y ello, a través de planeaciones didácticas innovadoras, estructuradas y complejas que se llevan a cabo en la práctica docente, para el cumplimiento del proceso educativo.

Para el caso que nos ocupa, nos centramos en una de las funciones sustantivas de las IES, que es la participación activa de los docentes y/o Facilitadores de la enseñanza - aprendizaje, al ser los garantes de la formación integral de los estudiantes y futuros profesionistas; este rol se significa al ser los responsables de "contribuir en las aulas a crear espacios dinámicos, creativos, facilitadores de los aprendizajes, promotores de valores de convivencia y ciudadanía" (UNESCO, 2016, p. 3) y que su actuar, a través de innovaciones y estrategias pedagógicas y metodológicas pertinentes, 
propicia los ambientes de aprendizaje necesarios, los procesos de evaluación imprescindibles y la generación de materiales acordes a las necesidades $y$ a el contexto del siglo XXI (SEP, 20I6), poniendo en el centro el proceso de desarrollo de los estudiantes.

En ese sentido, el papel de los docentes y/o facilitadores y la innovación educativa y estratégica que implementen, cobra un sentido sustancial para contribuir al logro de los retos y desafíos que plantea la Educación Superior del Siglo XXI, tal como lo asevera:

...la UNESCO (20I4) la innovación educativa es un acto deliberado y planificado de solución de problemas, que apunta a lograr mayor calidad en los aprendizajes de los estudiantes, superando el paradigma tradicional. Implica trascender el conocimiento academicista y pasar del aprendizaje pasivo del estudiante a una concepción donde el aprendizaje es interacción $y$ se construye entre todos (citado por UNESCO, 2016, p. 3).

Bajo dicho marco y asumiendo los retos y desafíos que nos compete como una institución educativa, presentamos la experiencia y resultados de la aplicación de la intervención pedagógica-metodológica, que se origina desde el reconocimiento de la Unidad de Competencia y objeto de estudio del programa de la Experiencia Educativa (EE) de AFEL ¿Cómo aplicar la Estadística en proyectos de investigación? con el objetivo de fomentar el desarrollo del pensamiento estadístico y científico en los estudiantes, a través de la aplicación de técnicas y herramientas estadísticas a proyectos de investigación diversos y reales, con actitud ética y crítica para la resolución de problemas similares a los que se enfrentarán en el mercado laboral, y a través del diseño de una planeación didáctica innovadora insertada en las plataformas virtuales EMINUS 4 y Teams.

De ahí que se diseñaron estrategias metodológicas innovadoras para ser aplicadas en el proceso educativo de dicha $\mathrm{EE}$, con la finalidad principal de fortalecer la formación integral de los estudiantes y que dichas estrategias contribuyeran significativamente a su perfil de egreso y por ende, a su inserción al campo laboral con las competencias requeridas en su profesión.

\section{DesarRollo}

Para llevar a cabo el diseño de las estrategias y recursos innovadores a ser implementados, identificamos dos problemáticas principales, por un lado, el hecho de que los estudiantes, a pesar de haber estudiado cursos de matemáticas y estadística, desde el nivel básico hasta bachillerato, no han logrado desarrollar la habilidad del Pensamiento Estadístico, más allá de la mera aplicación de fórmulas (Del Callejo et al, 2020), es decir, no hay evidencias tangibles de que sean capaces de demostrar competencias de análisis de situaciones del entorno, argumentación y razonamiento matemático, competencias cognitivas que se requieren para el desarrollo de dicho pensamiento estadístico; además, estos saberes conforman el núcleo de conocimientos básicos que permiten "El desarrollo de las capacidades de pensamiento crítico, análisis, razonamiento lógico y argumentación" (SEP, 2016, p. 15). 
Como evidencia de ello, en los resultados del Programa para la Evaluación Internacional de Alumnos de la OCDE (PISA) del 2018, se resalta que "Alrededor del $44 \%$ de los estudiantes en México alcanzó el nivel 2 o superior en matemáticas. Estos estudiantes pueden interpretar y reconocer, sin instrucciones directas, cómo se puede representar matemáticamente una situación simple" (OCDE, 2018, p. 4). Asimismo, "alrededor del I\% obtuvo un nivel de competencia 5 o superior. ... Estos estudiantes pueden modelar situaciones complejas matemáticamente y pueden seleccionar, comparar y evaluar estrategias apropiadas de resolución de problemas para tratar con ellos" (OCDE, 20I8, p.4).

De ahí que la metodología y estrategias implementadas se enfocaron en tratar de lograr un desempeño académico idóneo de los estudiantes, desde el objeto de estudio de la EE, a fin de que los estudiantes sean capaces de recolectar datos e información, interpretar y presentar con claridad resultados e inferencias con el uso pertinente $y$ eficaz de la Estadística, vitales no solo para investigadores o profesionales de la Estadística, sino para los ciudadanos en general (Franklin et al, 20I5 y Weiland, 20I7).

Como segunda problemática identificada es que originalmente la EE se diseñó en modalidad presencial y por la pandemia del Covid 19, se tuvo que reestructurar en modalidad virtual, a través de las plataformas de EMINUS 4 (Sistema de Educación Distribuida) y Teams, creando una nueva planeación didáctica, nuevos ambientes virtuales de enseñanza-aprendizaje, estableciendo el cuándo, cómo, dónde y con qué se implementaría el proceso educativo, para garantizar que los estudiantes alcancen las competencias teóricas, heurísticas y axiológicas establecidas en el programa de estudios.

\section{CONTEXTO DE LA APLICACIÓN}

La EE ¿Cómo aplicar la Estadística en proyectos de investigación? se desarrolló en periodo intersemestral (6 créditos); pertenece al Área de Formación de Elección Libre; contó con 19 estudiantes inscritos: 6 del área de Ciencias de la Salud, 2 de Biológico-Agropecuarias, 7 de la Técnica, 2 de Humanidades y 2 del Económico-Administrativo. La planeación flexible tiene como fundamento teórico y alcance la Unidad de Competencia planteada en el Programa de estudios, la filosofía del MEl y el enfoque por competencias, la complejidad, el uso de las Tecnologías de la Información y Comunicación (TIC), la técnica del Aprendizaje Basado en Problemas (ABP), el Aprendizaje Cooperativo, la Guía del Docente y la Ética aplicada como eje transversal (UVa, 2020).

Recuperamos nuestras experiencias docentes anteriores, las características y necesidades de las nuevas generaciones de estudiantes, las posibles ausencias en temas estadísticos por provenir de diferentes formaciones disciplinarias, lo que estimuló la necesidad de diseñar estrategias innovadoras para la resolución de un problema de investigación y/o proyecto de tesis real, aplicando saberes de la metodología estadística de manera correcta y ética, desarrollando habilidades, destrezas y valores en procesos formales de investigación y utilizando recursos y medios tecnológicos para promover la curiosidad en los estudiantes $\mathrm{y}$ fomentar el aprendizaje autónomo y el logro de las competencias planeadas. 


\section{DESCRIPCIÓN DE LA INNOVACIÓN EDUCATIVA}

Partimos de la necesidad de romper con los esquemas tradicionales para "provocar cambios en las prácticas educativas vigentes: en las estructuras, mentalidades y procederes dentro de un proceso educativo, con el fin de promover aprendizajes significativos" (UVb, 2020, p. s/n). Por ello, nuestra planeación didáctica (flexible, de acuerdo con los avances de los estudiantes), tiene dos miradas: primero, la claridad de que las acciones que realizaran los estudiantes -ejecutores de su propio aprendizaje-, giren alrededor del problema de investigación, como un todo complejo, que se reflejara en su proyecto integrador final; y, segundo, como guía para el Facilitador en el desarrollo, seguimiento y conclusión del curso: qué, cuándo, cómo y temporalidad (García, 20I4).

Para ello, las estrategias secuenciales o por dominios fueron congruentes y pertinentes tanto con la Unidad de Competencia como con el objetivo de la EE para que estas fueran acordes con el desempeño deseado del estudiante, como parte de su proceso de construcción del aprendizaje, es decir, de aprender a conocer, a hacer, a convivir y a ser (Delors, 1996) de manera autorregulada. Las estrategias teórico metodológicas innovadoras implementadas son:

I. MEl y enfoque por competencias. Basamos el proceso a partir del fundamento, filosofía, fines y ejes del MEl tanto en la planificación como en la ejecución, articulamos la Unidad de Competencia (comprensión y aplicación de la Estadística), los saberes $y$ actividades de aprendizaje en congruencia con las evidencias de desempeño y el producto final esperado.

2. Adaptación de la metodología basada en tres dominios instruccionales -subcompetencias-. Propuestos por
Garfield et al (2003), que implican niveles de complejidad y logro de objetivos de desempeño por cada dominio: Alfabetización estadística, Razonamiento estadístico y Pensamiento estadístico. De acuerdo con Del Callejo et al (2020):

... la finalidad de esta intervención pedagógica es provocar que los estudiantes desarrollen el pensamiento estadístico, a través de un aprendizaje gradual, partir de los conceptos y simbología básicos de la estadística (alfabetización estadística), deducir el porqué de la aplicación de la estadística en el uso y proceso de la información (razonamiento estadístico) y lograr que sean capaces de evidenciar su desempeño a través de la demostración y aplicación de los conocimientos y habilidades necesarias para analizar e interpretar y proponer alternativas de solución a problemáticas de su entorno, generándose aún más preguntas a futuro (pensamiento estadístico). (p.199)

3. Aplicación de la técnica didáctica Aprendizaje basado en problemas. Esta técnica, unida al enfoque por competencias, permite visualizar cómo los estudiantes van construyendo su aprendizaje, a partir de su propia elección del problema a investigar, estableciendo hipótesis estadísticas, indagando y aplicando los procedimientos y herramientas estadísticas a utilizar para resolverlo, recuperando saberes previos y utilizando el pensamiento crítico para interpretar con ética los resultados sobre el objeto de estudio, utilizando la metodología estadística para la explicación del fenómeno de la realidad, es decir, aprendiendo 
por descubrimiento (UVc, s/f) en lo individual y en pequeños grupos.

4. Mensaje de Bienvenida, en EMINUS 4 y reunión inicial por Teams. Este primer acercamiento con los estudiantes define $y$ abre los canales de comunicación a fin de que los estudiantes se sientan en confianza de que el facilitador los acompañará en una realidad virtual durante la construcción de sus aprendizajes. Además, en esa primera sesión, se analiza el programa de la EE, su estructura, contenidos, calendarización de las actividades y procedimiento de evaluación del curso e invita a los estudiantes a explorar la plataforma.

5. Cuestionario diagnóstico inicial y final (plataforma forms de Google). Este instrumento pretende recabar información sobre: Datos generales, expectativas y motivos de elegir esta EE, conocimientos previos sobre la Estadística y la Ética en la Investigación, acceso y disponibilidad de equipo de cómputo e internet con la finalidad de obtener información sobre necesidades a considerar, así como el impacto-resultadosconveniencia-pertinencia de la aplicación de las estrategias innovadoras.

6. Definición del Rol activo del estudiante. Desde el inicio, el estudiante reflexiona sobre su entorno y elige por sí mismo, un problema de investigación real (objeto de estudio) ya sea de interés personal y/o profesional, para inducir su motivación, su creatividad y curiosidad por descubrir respuestas.

7. Retroalimentaciones. Consideramos esta acción permanente como aquel proceso sistemático para atender dudas y comentarios y emitir un juicio fundamentado que contribuya a la comprensión del "objeto de estudio" y toma de decisiones. Éstas se realizan los tres primeros días para comunicar los aciertos, las debilidades y áreas de mejora. La primera se efectúa a través de una sesión sincrónica por TEAMS, donde se recrea un ejemplo de un estudiante ficticio que reúne los errores más comunes presentados por los estudiantes. El Facilitador explica dónde está el error y como solventarlo.

8. Dinámica de trabajo. Redacción de indicaciones claras y precisas para 5 actividades individuales donde el estudiante visualiza el módulo y accede: al podcast (Puntadas al azar); al canal de Youtube (Diana Del Callejo); a las notas de clase en formato PDF, disponibles desde EMINUS 4 y TEAMS; a un mini-video -contenido transversalsobre Ética en la Investigación; y a la actualización en las técnicas estadísticas y los comandos en el software libre R-Studio. Además, se plantean dos actividades grupales, donde partiendo de su trabajo individual y con una Rúbrica de evaluación (Tabla I) proporcionada y explicada por el Facilitador en sesión sincrónica el estudiante I, evalúa/retroalimenta al estudiante 2 y viceversa. Dicha retroalimentación se entrega al compañero a través de Teams y con base en ello, el estudiante realiza modificaciones $y$ finalmente es retroalimentada por el Facilitador. Este proceso se basa en la metodología de Aprendizaje cooperativo'. 


\section{Tabla I}

Rúbrica de evaluación (Aprendizaje cooperativo).

\begin{tabular}{|c|c|c|}
\hline CONCEPTO & CALIFICACIÓN & OBSERVACIONES \\
\hline \multicolumn{3}{|c|}{ FORMA (Valoración binaria: si o no). } \\
\hline \multicolumn{3}{|l|}{ El trabajo cumple con el tipo de letra establecido. } \\
\hline \multicolumn{3}{|l|}{ El trabajo respeta el interlineado establecido. } \\
\hline \multicolumn{3}{|c|}{ El nombre del estudiante se encuentra a alineado a la derecha. } \\
\hline \multicolumn{3}{|l|}{ En el nombre del estudiante hay una nota al pie. } \\
\hline \multicolumn{3}{|c|}{ FONDO (Valoración de I al I0) } \\
\hline \multicolumn{3}{|l|}{ El trabajo cuenta con un objetivo claro. } \\
\hline \multicolumn{3}{|c|}{ En la metodología se dice cuántos individuos y quiénes son. } \\
\hline \multicolumn{3}{|l|}{ En la metodología se dice cuántas variables y cuales son. } \\
\hline \multicolumn{3}{|c|}{ En la metodología se describe el tipo de variables o su tipo de escala. } \\
\hline \multicolumn{3}{|c|}{ En la metodología se describe el proceso estadístico que se siguió. } \\
\hline \multicolumn{3}{|c|}{ La metodología descrita es la que aplica para los datos. } \\
\hline \multicolumn{3}{|l|}{ La metodología se escribe en pasado. } \\
\hline \multicolumn{3}{|c|}{ En los resultados, se describe con claridad los hallazgos. } \\
\hline \multicolumn{3}{|l|}{ Los resultados coinciden con la metodología descrita. } \\
\hline \multicolumn{3}{|c|}{$\begin{array}{l}\text { Si es que hay gráficos, estos cuentan con los lineamientos gráficos siguientes: } \\
\text { - Título que refleje claramente lo que describe el gráfico. } \\
\text { - Nombre de las categorías incluidas (Eje X). Si es pastel, asegurar que las } \\
\text { categorías son visibles. } \\
\text { - Frecuencias o frecuencias relativas (Eje Y). Si es pastel asegurar que queda } \\
\text { claro si son frecuencias, o frecuencias relativas. } \\
\text { - Fuente de la que se obtuvieron los datos. }\end{array}$} \\
\hline \multicolumn{3}{|l|}{ Se hace alusión del gráfico o tabla en los resultados. } \\
\hline \multicolumn{3}{|l|}{ Los gráficos o tablas están numerados. } \\
\hline Los resultados están completos. & & \\
\hline
\end{tabular}

9. Proyecto integrador final. La evidencia final de aprendizaje tiene la intención de presentar los resultados del problema investigado y se presenta en forma de artículo para revista científica (Figura I), en formato IMRyD (Introducción, Métodos, Resultados y Discusión), lo que otorga un plus innovador a esta EE. Los estudiantes cuentan con la asesoría previa del Facilitador sobre la estructura y elementos a considerar y a través de una dinámica en parejas y en sesión sincrónica, el grupo I retroalimenta al grupo 2 y viceversa.

Posterior a ello, el Facilitador hace su retroalimentación final. Este producto consolida y permite identificar los saberes alcanzados por los estudiantes durante y al final del curso (Unidad de competencia), así como el impacto de las estrategias aplicadas. 


\section{Figura I}

Ejemplo de trabajo final en formato IMRyD
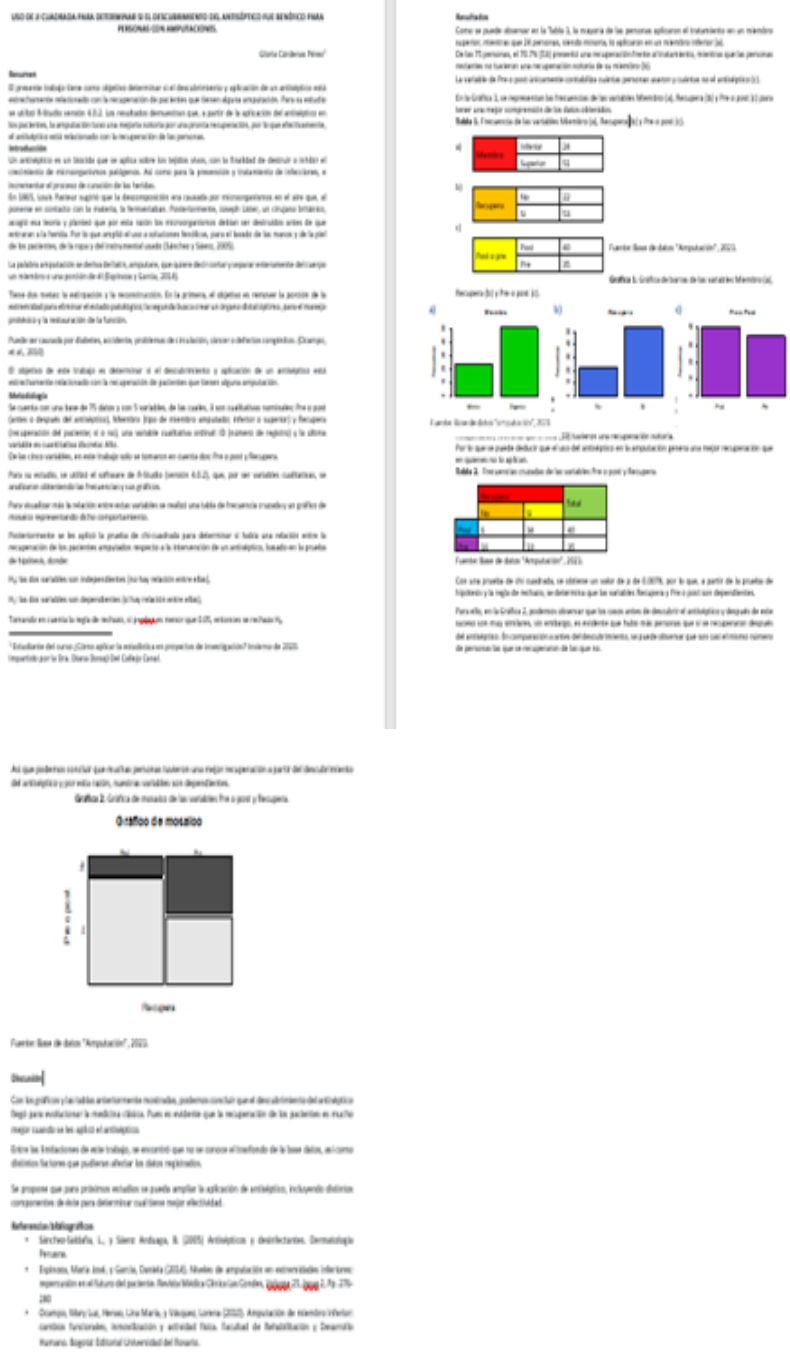

10. Proceso de evaluación. Nos cuestionamos desde la planeación el qué, para qué y el cómo evaluar.

Consideramos como estrategia la Evaluación

formativa, pues ésta permite visualizar el desempeño de los estudiantes y la complejidad del aprendizaje, otorgando igual relevancia a las evidencias finales como al proceso que dio paso a alcanzar el Pensamiento estadístico. Para realizar este tipo de evaluación, fue necesario establecer los criterios de evaluación (Tabla 2), conocido por los estudiantes desde el inicio del curso.

\section{Tabla 2}

Criterios de evaluación por dominio alcanzado
PUNTAJES

\begin{tabular}{|c|c|}
\hline & EVALUACIÓN/DOMINIOS \\
\hline $\begin{array}{l}\text { Calificación de } 5 \\
\text { (no acreditado) }\end{array}$ & $\begin{array}{l}\text { Si el estudiante no entrega los avances durante el } \\
\text { curso y no logra aplicar una técnica estadística } \\
\text { correcta para resolver su pregunta de } \\
\text { investigación y tampoco logra explicarla de } \\
\text { manera escrita y oral. }\end{array}$ \\
\hline Calificación 6 a 7 & $\begin{array}{l}\text { Si el estudiante logra aplicar la técnica estadística } \\
\text { correcta para resolver su pregunta de } \\
\text { investigación, pero tiene dificultades para } \\
\text { argumentar el procedimiento estadístico, de } \\
\text { escribir y explicar oralmente sus hallazgos, se } \\
\text { asume el logro de Alfabetización estadística. }\end{array}$ \\
\hline Calificación 8 & $\begin{array}{l}\text { Si el estudiante logra aplicar la técnica estadística } \\
\text { correcta para resolver su pregunta de } \\
\text { investigación, explica con dificultad el porqué de } \\
\text { ésta en la resolución de su pregunta, pero sin } \\
\text { llegar a hacer juicios de valor basados en } \\
\text { criterios específicos, con organización, con } \\
\text { lenguaje adecuado, etc., se asume que está en la } \\
\text { etapa de Razonamiento Estadístico. }\end{array}$ \\
\hline $\begin{array}{l}\text { Calificación } 9 \text { a } \\
10\end{array}$ & $\begin{array}{l}\text { Si el estudiante logra aplicar la técnica estadística } \\
\text { correcta para resolver su pregunta de } \\
\text { investigación, sin dificultades para escribir y } \\
\text { explicar oralmente sus hallazgos, con } \\
\text { argumentación, interpretación y valoración de } \\
\text { qué información es pertinente para la } \\
\text { problemática en concreto, con preguntas } \\
\text { avanzadas sobre el uso de la Estadística, con } \\
\text { coherencia y claridad, se asume que está en la } \\
\text { etapa Pensamiento Estadístico. }\end{array}$ \\
\hline
\end{tabular}

\section{MEDIOS Y RECURSOS TECNOLÓGICOS DE APOYO AL APRENDIZAJE}

Llevar a cabo el proceso educativo en la modalidad en línea, nos permitió el uso de las TIC y el internet, para ello, diseñamos e implementamos medios y recursos didácticos innovadores, basados en el enfoque pedagógico de Flipped Learning o Aprendizaje invertido, como apoyo y orientación a la exposición de ciertos contenidos, cuya fortaleza es que el estudiante 
tiene la posibilidad de controlar "aprendizaje autónomo", el tiempo, lugar, ruta y ritmo, sin que con ello, pierda la posibilidad de que en las sesiones virtuales con el Facilitador, cuente con supervisión, asesoría y aclaración de dudas. Esto en sí es un reto tanto para el docente como para el estudiante, implica: un cambio en la cultura de la enseñanza-aprendizaje; requiere investigación para definir qué hacer, qué seleccionar y cómo utilizar los medios y recursos para propiciar el aprendizaje; y considerar la brecha digital entre los estudiantes, ya que no todos se pueden allegar los recursos necesarios para enfrentar dicho reto.

Para tal efecto, nos cuestionamos: ¿Qué estrategias y medios didácticos son necesarias y factibles de implementar para apoyar a los estudiantes universitarios, de diversos programas educativos, a que desarrollen y apliquen el Pensamiento Estadístico? Reflexionamos en torno a decidir cuáles, cómo y cuándo utilizar estos recursos de acuerdo con el contenido a revisar, los objetivos que esperamos alcanzar, los contenidos que requieren ser ampliados o reforzados para garantizar un aprendizaje significativo (García, 20I4), entre otros.

Para ello, implementamos en ambientes sincrónicos y asincrónicos lo siguiente:

I. Chat permanente en TEAMS: este recurso y canal de comunicación en línea permite realizar las retroalimentaciones, responder dudas $\mathrm{y}$ atender comentarios de los estudiantes, creando un ambiente adecuado para el aprendizaje, generando interrelaciones y confianza (SEP, 20I6) en una realidad virtual.

2. Podcasts educativos para contenidos de mayor complejidad: De acuerdo con Solano y Sánchez, son "un medio didáctico que supone la existencia de un archivo sonoro con contenidos educativos y que ha sido creado a partir de un proceso de planificación didáctica” (2010, p. 128). Los contenidos que utilizamos son abiertos, se exponen con un lenguaje sencillo sobre temas estadísticos y los estudiantes pueden observarlo cuantas veces lo requieran (Figura 2, Figura 3 y Figura 4), disponible en Spotify, Anchor, Google Podcast, Apple Podcast, etc. https://anchor.fm/dianadelcallejo

\section{Figura 2}

Ejemplo de preparación de un Podcast: Limpiar una tabla de Datos

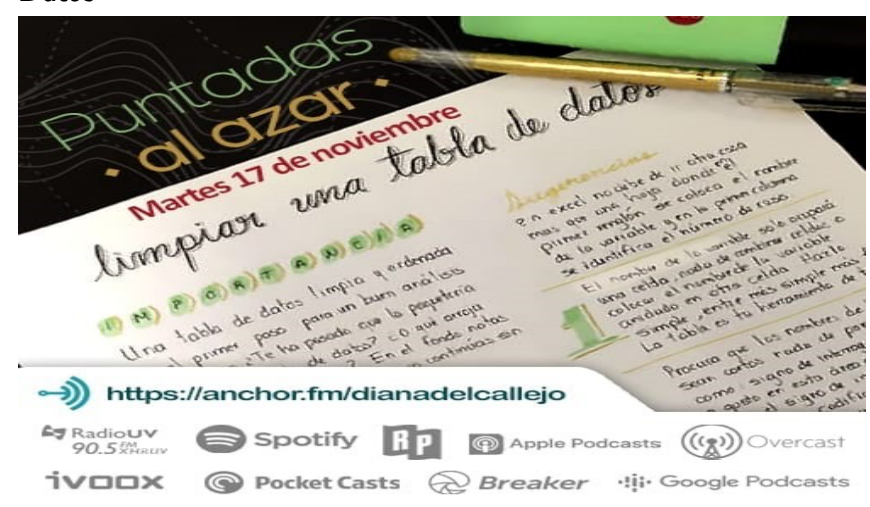

\section{Figura 3}

Ejemplo de preparación de un Podcast: Prueba de hipótesis

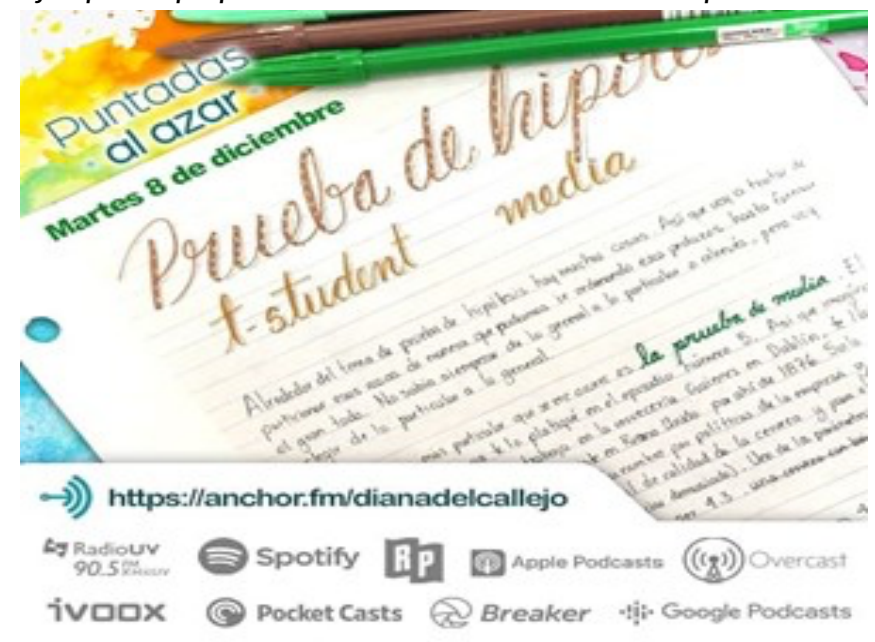




\section{Figura 4}

Ejemplo de preparación de un Podcast: Prueba T-student

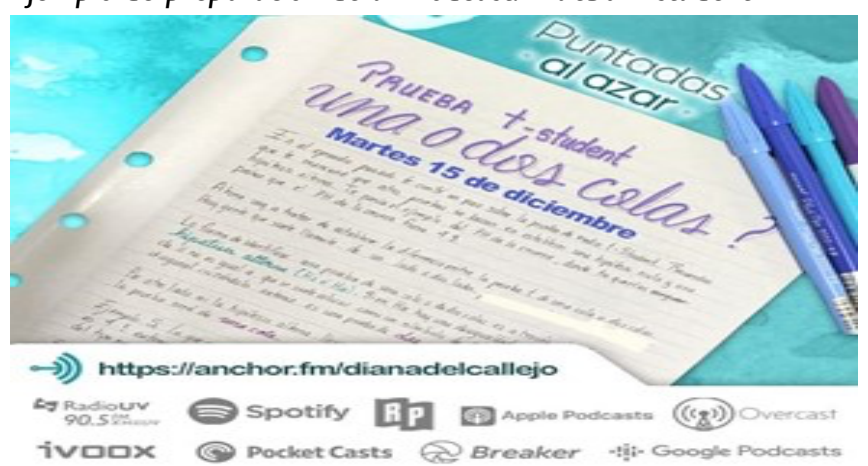

3. Notas de clase: herramienta visual, para hacer un resumen sintetizado de lo que se escucha en el podcast (Figura 5, Figura 6) agregando claves del proceso estadístico: fórmulas, gráficos o tablas para fortalecer los conocimientos y habilidades sobre la Estadística.

\section{Figura 5}

Ejemplo de notas de clase: Medidas de tendencia central

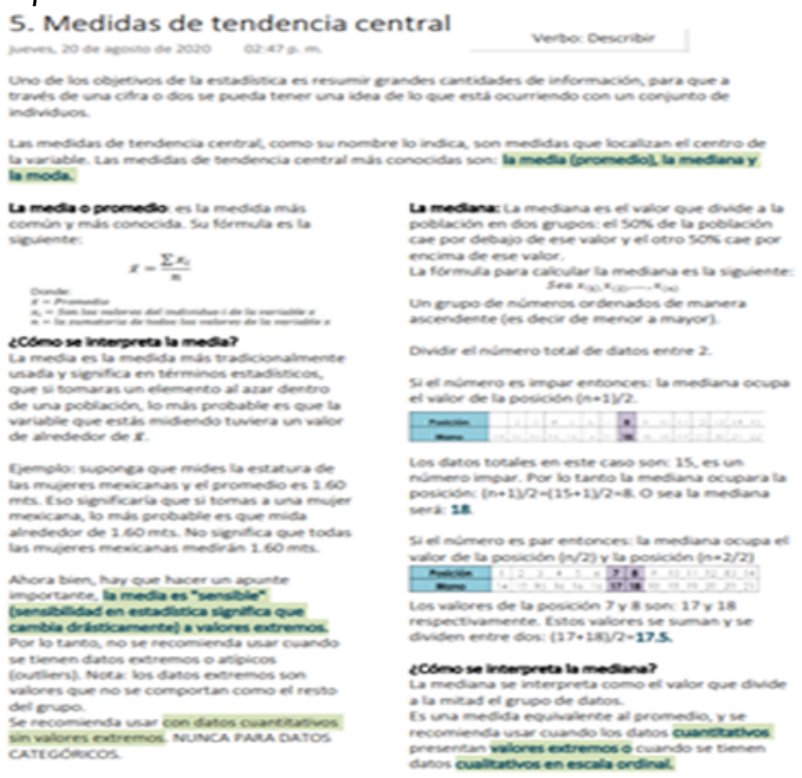

\section{Figura 6}

Ejemplo de notas de clase: Medidas de dispersión

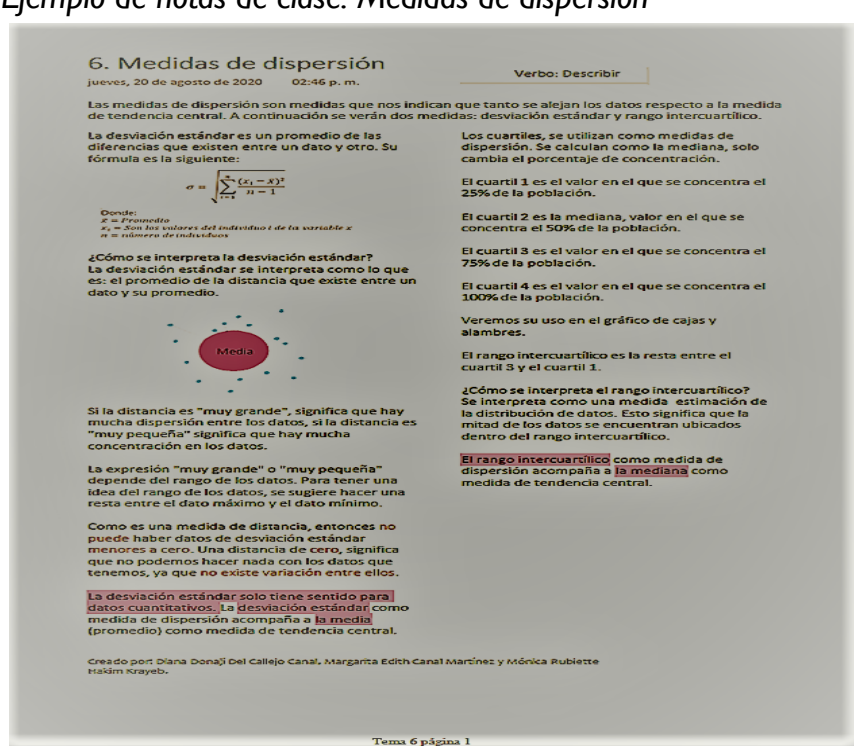

4. Mini-videos educativos de corta duración: de la Fuente et al. (20I3) definen "al mini vídeo como un vídeo de corta duración que constituye un material didáctico de tipo tecnológico para transmitir una determinada información que ayude a consolidar cierto aprendizaje" (p.I80). Utilizamos esta herramienta visual y auditiva para reforzar de forma atractiva y dinámica los saberes de la EE y guiar al estudiante en el proceso de comprensión del uso pertinente de la estadística en sus proyectos de investigación. La elaboración de los Mini-vídeos (Figura 7, Figura 8), tienen presente las siguientes finalidades: el propósito educativo, que es lograr y/o reforzar el aprendizaje y el promover el interés del estudiante; un soporte material, que en este caso es el programa de la $\mathrm{EE}$; un contenido particular relacionado con el tema a tratar; una representación simbólica y atractiva de la información. Dichos mini-vídeos se encuentran disponibles en:

https://www.youtube.com/channel/UCaEtbM2Pxp

\section{CFpQRmnRTaaqQ/videos}




\section{Figura 7}

Ejemplo de un mini-video: Histograma de largo de pétalo

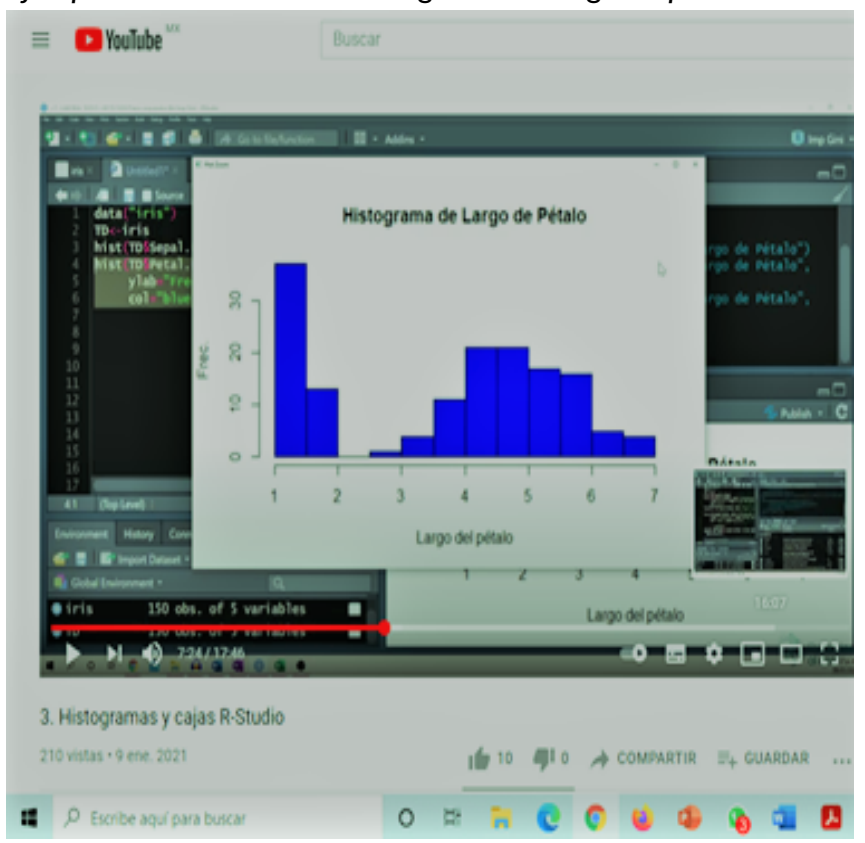

\section{Figura 8}

Ejemplo de un mini-vídeo: Pearson y diagrama de dispersión en $R$ Studio

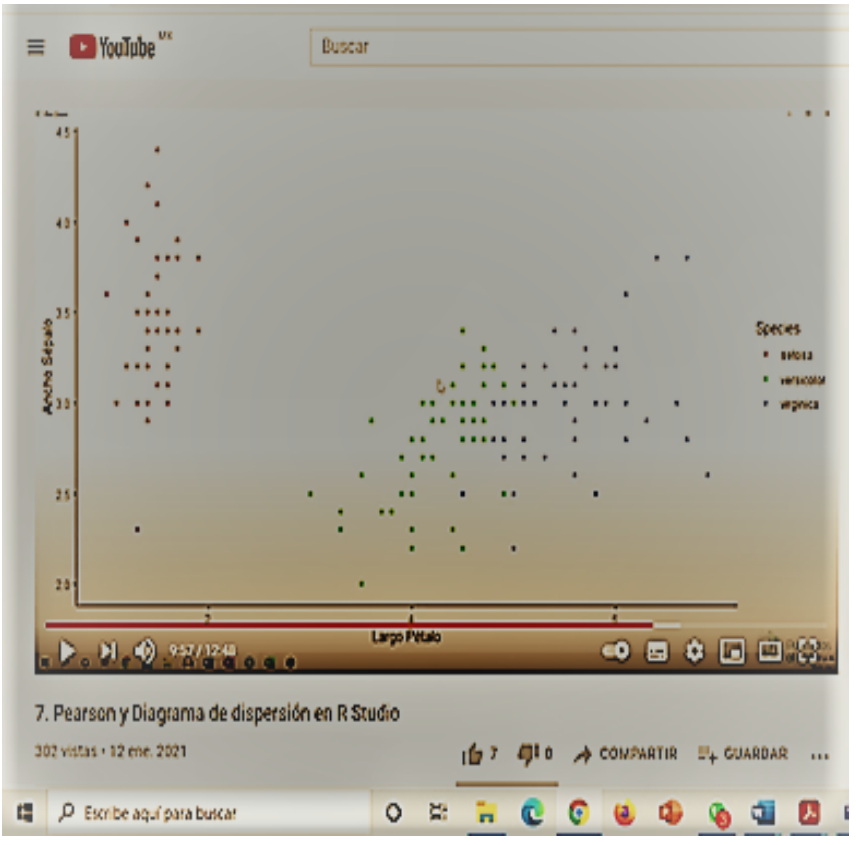

(Figura 9, Figura 10). Incluye una consola, editor de sintaxis que apoya la ejecución de código, así como herramientas para el trazado, la depuración y la gestión del espacio de trabajo (Equipo RStudio, 2020).

\section{Figura 9}

\section{Matriz de correlaciones en R-Studio}

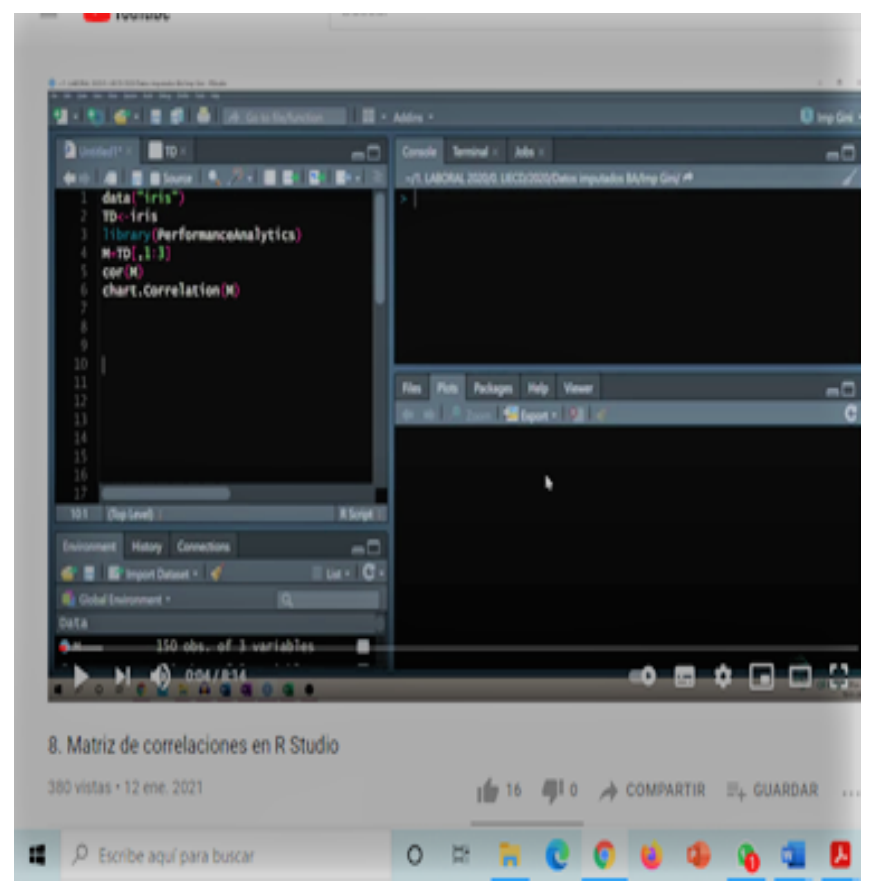

\section{Figura 10}

Exportar una gráfica de R-Studio

5. Software libre R-Studio (RStudio Team, 2020): dicho software es un entorno de desarrollo integrado (IDE) para el lenguaje de programación $R$, es utilizado para la computación estadística y gráficos 


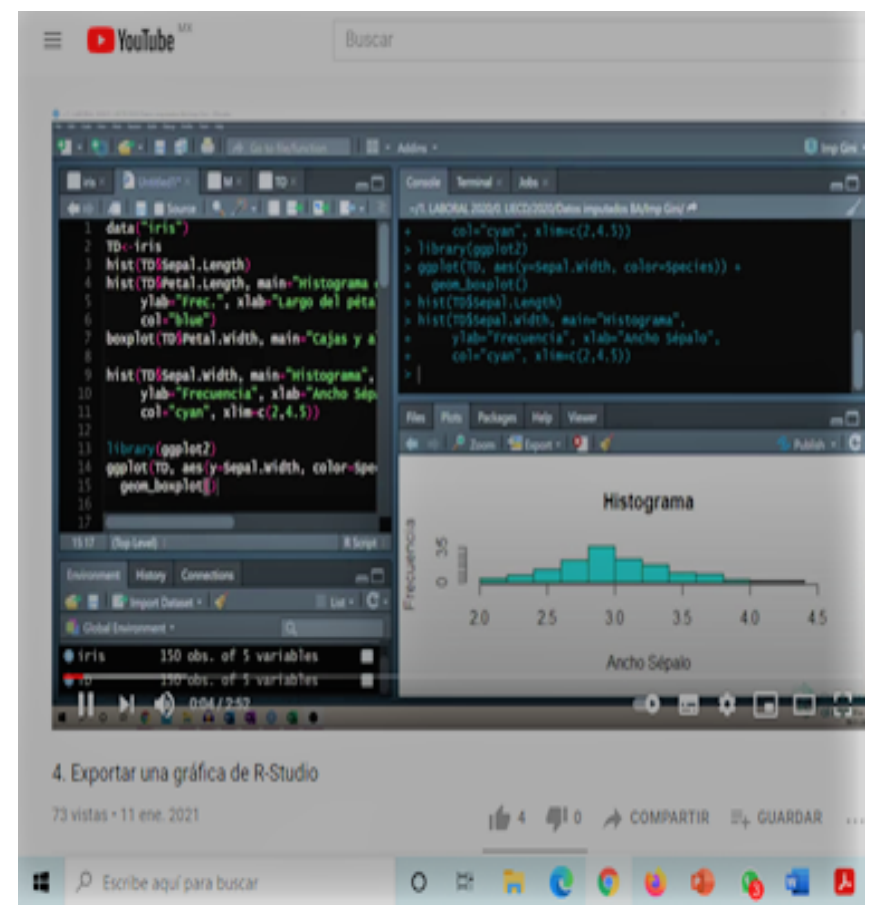

6. Mini-video para contenido transversal: por la experiencia de varios años impartiendo docencia, hemos detectado que los estudiantes desconocen los procedimientos para citar a los autores que consultan, utilizando solamente el copiar y pegar.

Además, consideramos pertinente darles a conocer algunos elementos clave sobre cómo la Ética se aplica a los proyectos de investigación respetando las normas y principios que deben ser observados en el campo de la ciencia (Figura II).

Ello con la finalidad de que comprendan la importancia de cuidar la reputación profesional sin que se afecte sus posibilidades laborales futuras (Soto, 20I2). Disponible en https://Idrv.ms/v/s!Asdi5hDQhsGGg3xLfBPSdsYEL $\mathrm{mSr} ? \mathrm{e}=\mathrm{AjPi} 2 \mathrm{Q}$

\section{Figura I I}

Mini-vídeo ética en la Investigación

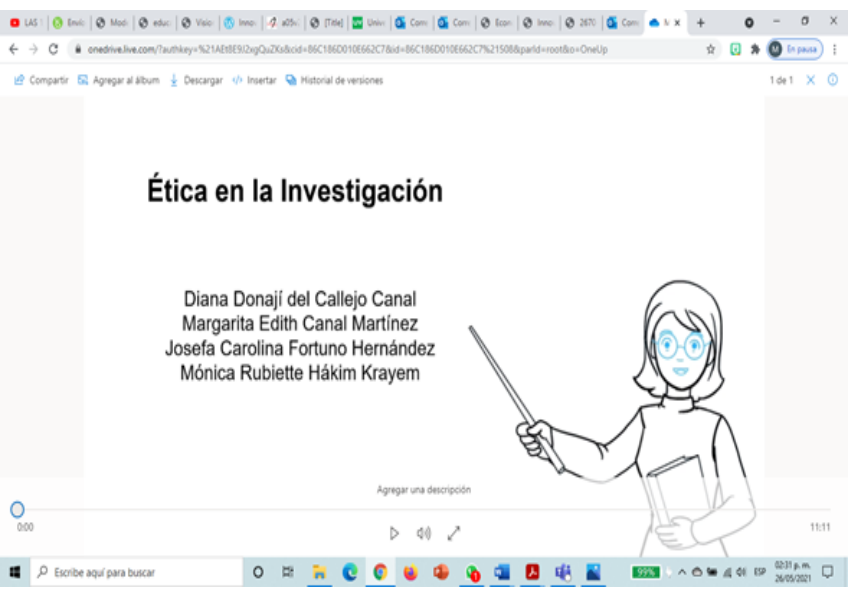

\section{RESULTADOS Y CONCLUSIONES}

Existen distintas formas de definir la innovación, sin embargo, se coincide en que no se trata de una tarea sencilla o de mejora, sino de romper esquemas que den paso a la evolución o transformación del proceso de enseñar y aprender, por ello, reconocemos que la implementación realizada nos permitió innovar en el proceso educativo de la EE ¿Cómo aplicar la Estadística en proyectos de investigación?

Con la diversidad de programas educativos de procedencia y formaciones de los estudiantes, las estrategias innovadoras aplicadas y el objetivo de la EE, se cumplió significativamente en las categorías de suficiencia, pertinencia y congruencia, ya que los estudiantes evidenciaron el desarrollo de competencias disciplinares, específicas y transversales y sus respuestas sobre la metodología, medios y recursos aplicados, favorecieron su formación integral y, por ende, benefició en su perfil de Egreso.

Del total de los estudiantes, el $89 \%$ acreditaron la EE. El 47\% desarrolló el Pensamiento estadístico, un 37\% el Razonamiento estadístico, un 5\% alcanzó la Alfabetización estadística, de acuerdo con los criterios de evaluación establecidos. Por otro lado, el II\% de los estudiantes no acreditaron la $\mathrm{EE}$, dado que no 
entregaron las evidencias de sus actividades, durante $y$ al final del curso, debido, en parte, a que algunos tramitaron Baja de la EE.

Respecto al Cuestionario inicial y final. Al termino del curso, un $94 \%$ asociaron a la estadística, con: información, analizar, realidad, interpretar y ayuda, palabras que al inicio del curso no se mencionaron. En cuanto al tema transversal de la Ética aplicada a la investigación, al inicio aparecen las palabras persona, importante, beneficio; al final se identifican las palabras trabajo, correcta, respetar y resultados.

Las retroalimentaciones fueron sobresalientes, oportunas y crearon un ambiente de confianza con y entre los estudiantes. Se evidenció, también, que los medios y recursos utilizados resultaron ser herramientas innovadoras para el logro de la Unidad de competencia, además de ser interactivos y motivantes, permitiendo a los estudiantes un aprendizaje autónomo -posibilidad de controlar el tiempo, lugar, ruta y ritmo-, en ambientes asincrónicos.

Consideramos, además, que en la medida que el uso de las TIC permee el ámbito educativo, éstas serán consideradas cada vez más, como una herramienta de apoyo para generar aprendizajes -saberes heurísticos- $y$ dejaremos de sólo acceder y transmitir información, error que sigue englobando a la educación tradicional (Mestres, 2008). Por ello, los recursos y medios didácticos diseñados y aplicados con el soporte tecnológico actual, como los podcasts y mini-videos utilizados, resultaron ser una herramienta relevante como complemento del aprendizaje de los estudiantes.

\section{Tabla 3}

Algunas evidencias textuales de estudiantes sobre la EE y las estrategias aplicadas.
"[...]La verdad es que los podcast y los vídeos me ayudaron bastante, de hecho los vídeos me sirvieron más que el librito de comandos que de por si es muy bueno, pero verlo así de que yo voy a la parte del vídeo, es como estar en una clase y pues así te puedes ayudar, igual alguna duda de teoría lo veía o volvía a escuchar el podcast, le ponía pausa, lo regresaba y todo era a mi tiempo no, y yo creo que eso también me podía hacer sentir más en la clase en cualquier momento que yo hiciera la tarea." (Karen Josseline Mota Prescenda, estudiante de Ingeniería Mecánica).

"[...] Los materiales que usted nos dio fueron de gran ayuda, fueron muy útiles, realmente los vídeos, es como si usted nos llevara de la mano para ir haciendo todo en el software de RStudio y algo que me gustó mucho de los podcast es que también nos introducía con un poquito de historia, siento que es muy importante eso [...]" (José Ferrer Hernández Cartagena, estudiante de Medicina).

Consideramos que, de acuerdo con nuestra experiencia docente, se destacan varios niveles de impacto de las practicas innovadoras aplicadas en la $\mathrm{EE}$ ¿Cómo aplicar la estadística en proyectos de investigación? Retomando algunas conclusiones de Jerez y Silva (2017) en Gonzáles y Cruzat (2019), obtuvimos un logro en la docencia, al obtener resultados de aprendizaje de un $89 \%$ de aprobación (nivel I); la participación entusiasta y motivadora de los involucrados en el proceso innovativo, los estudiantes expresaron su opinión positiva sobre el proceso educativo experimentado (Tabla 3), encontraron significatividad asociada al aprendizaje y utilidad de la innovación entre ellos, al ser también retroalimentadores de los trabajos (nivel 2); trascendencia en la cotidianidad formativa (nivel 3) en tres aspectos que mencionados por Jerez y Silva (2017 como se citó en Gonzáles y Cruzat 2019):

... Impacto en la cotidianidad formativa. Este impacto se refiere a tres focos: -Rutinas claves: Impacto en las acciones de docentes $y$ estudiantes al interior de procesos de enseñanza aprendizaje. - Cambios formales: 
Cambio en los recursos, herramientas y estructuras asociadas a los procesos de enseñanza aprendizaje. - Uso de un lenguaje pedagógico: Uso del lenguaje para establecer significados, adopción de conceptos claves que pasan a ser propios de la comunidad académica (p. II2).

Por tanto, concluimos que las estrategias implementadas son factibles de aplicarse a otras EE, a través de una planeación y adecuación de los medios y recursos a las competencias deseadas, para favorecer la formación integral de los estudiantes, siempre y cuando los Facilitadores asuman la responsabilidad que requieren los desafíos educativos actuales; ello permitirá además hacer comparaciones del impacto.

\section{REFERENCIAS}

Asociación Nacional de Universidades e Instituciones de Educación Superior [ANUIES] (20I8). Visión y acción 2030. Propuesta de la ANUIES para renovar la educación superior en México. https://visionyaccion2030.anuies.mx/Vision_accio n2030.pdf

de la Fuente Sánchez, D., Hernández Solís, M., y Pra Martos, I. (20I3). El Mini video como Recurso Didáctico en el Aprendizaje de materias cuantitativas. RIED. Revista Iberoamericana de Educación a Distancia, I6(2), pp. I77-192. Asociación Iberoamericana de Educación Superior a Distancia Madrid, Organismo Internacional. https://www.redalyc.org/pdf/33 |4/33 |429869008 .pdf

Del Callejo-Canal, D.; Canal-Martínez, M. y Hákim, R. (2020). Desarrollo del pensamiento estadístico en estudiantes de nivel superior a través de una Experiencia Educativa. Revista Educación Matemática. 32(2). https://www.revistaeducacion-matematica.org.mx/revista/volumen32-numero-2-agosto-20201

Delors, J. (1996). Los cuatro pilares de la educación. En la educación encierra un tesoro. Informe a la UNESCO de la Comisión internacional sobre la
Educación para el siglo XXI. Madrid, España: Santillana/UNESCO. pp. $91-103$.

Franklin, C. A., Bargagliotti, A. E., Case, C. A., Kader, G. D., Scheaffer, R. L., y Spangler, D. A. (20I5). Statistical education of teachers (SET). www.amstat.org/education/SET/SET.pdf

García, M. (20I4). Uso instruccional del video didáctico. Revista de Investigación, 38 (8I) Pp. 4367. Universidad Pedagógica Experimental. Libertador Caracas, Venezuela.

Garfield, J., del Mas, R., y Chance, B. (2003). The Webbased ARTIST: Assessment Resource Tools for Improving Statistical Thinking. Paper Presented at the Annual Meeting of the American Educational Research Association, (April). https://publication/uuid/F567FA34-BCC6-49A2A6EC-8BC6BC88BFDC

González Castro, C. y Cruzat Arryagada, M. (2017). Innovación educativa: La experiencia de las carreras pedagógicas en la Universidad de Los Lagos, Chile. Educación XXVIII (55), septiembre 2019, pp. 103-122.

https://doi.org//0.18800/educacion.201902.005 
Mejía, M. R. (2000). La integración curricular una propuesta de transformación educativa. Ponencia presentada al $2^{\circ}$ Encuentro de Experiencias Educativas de Innovación, II y I2 de septiembre de 2000, Bucaramanga, Colombia. Fe y Alegría Universidad Autónoma de Bucaramanga.

Mestres, L. (2008). La alfabetización digital de los docentes. [Educaweb.com]. http://www.educaweb.com/noticia/2008/|2/0I/alf abetizacion-digital-docentes-3349/

Moreno O. Tiburcio. (20/2). La evaluación de competencias en educación.

http://www.scielo.org.mx/scielo.php?script=sci_a rttext\&pid=SI 665-109X20I 2000200010

Organizacion para la Cooperación y el Desarrollo Económicos [OCDE] (2018). Programa para la Evaluación Internacional de Alumnos. Resultados PISA.

http://www.oecd.org/pisa/publications/PISA2018 CN_MEX_Spanish.pdf

Organización de las Naciones Unidas para la Educación, la Ciencia y la Cultura [UNESCO]. (2016). Innovación Educativa. Serie Herramientas de apoyo para el trabajo docente. https://repasopcmasumet.files.wordpress.com/20 18/09/art-unesco-innovaciones-educativas-emetodologc3ada-4-innov-educ.pdf

RStudio Team (2020). RStudio: Integrated Development for R. RStudio, PBC, Boston, MA http://www.rstudio.com/

Secretaria de Educación Pública [SEP]. Modelo Educativo (2016). https:/www.gob.mx/cms/uploads/attachment/file/ | | 450|/Modelo_Educativo_2016.pdf

Solano F. Isabel y Sánchez V., Mª Mar. (2010). Aprendiendo en cualquier lugar: El Podcast Educativo. Pixel-Bit. Revista de Medios y Educación, (36). pp. 125-139. Universidad de Sevilla, España. https://www.redalyc.org/pdf/368/368I5I28010.p df

Soto, R. Armando. (20I2). El plagio y su impacto a nivel académico y profesional. Revista electrónica semestral. 2(I), Costa Rica. http://revistaebci.ucr.ac.cr

Universidad Veracruzana. (2020a) Pautas para la planeación flexible de Experiencias Educativas. México. https://www.uv.mx/desarrollocurricular/files/202 0/05/Planeación-Flexible-de-una-EE-DIE.pdf
Universidad Veracruzana. (2020b). Desarrollo curricular e Innovación educativa. México. https://www.uv.mx/desarrollocurricular/innovaci on-educatival

Universidad Veracruzana. (2020c). Coordinación de Aprendizaje basado en problemas. Introducción. https://www.uv.mx/abp/introduccion/

Weiland, T. (2017). Problematizing statistical literacy: An intersection of critical and statistical literacies. Educational Studies in Mathematics, 96(I), 33-47. https://doi.org//0.1007/s I0649017-9764-5 